Linguistique, littérature, didactique

177-178 | 2018

Langage oral à l'école maternelle. Étude d'un corpus

homogène

\title{
Analyses interactionnelle, syntaxique et régulatrice
}

Syntactic, interactive and regulative analysis

Nathalie Charvy

\section{(2) OpenEdition}

Journals

Édition électronique

URL : http://journals.openedition.org/pratiques/4103

DOI : 10.4000/pratiques.4103

ISSN : 2425-2042

Éditeur

Centre de recherche sur les médiations (CREM)

Référence électronique

Nathalie Charvy, "Analyses interactionnelle, syntaxique et régulatrice », Pratiques [En ligne],

177-178 | 2018, mis en ligne le 20 juillet 2018, consulté le 20 avril 2019. URL : http://

journals.openedition.org/pratiques/4103; DOI : 10.4000/pratiques.4103

Ce document a été généré automatiquement le 20 avril 2019

(C) Tous droits réservés 


\title{
Analyses interactionnelle, syntaxique et régulatrice
}

\author{
Syntactic, interactive and regulative analysis
}

Nathalie Charvy

1 Dans le contexte de la formation des enseignants, les contributions de chercheurs concernant l'apprentissage du langage oral en maternelle relèvent pour l'essentiel d'un champ théorique assez homogène - dont J. S. Bruner et L. Vygotski sont emblématiques. Il se trouve qu'en tant que formatrice d'enseignants, nous avons pris appui sur ces théoriciens pour mener une recherche sur l'influence des pratiques langagières enseignantes sur l'acquisition de la langue orale chez des élèves entre quatre et cinq ans, dans le cadre de narrations (Charvy, 2009). Les données du corpus CLEA sur lesquelles porte l'analyse ici présentée obligent à la confrontation entre cette première recherche et une approche étroitement dépendante du corpus en question. Cette étude comparative permet non seulement le questionnement d'un parcours de recherche mais aussi l'ouverture à d'autres champs théoriques, à d'autres modalités interactionnelles. Le travail proposé par P. Péroz (2015) vient interroger «une pratique scientifique stabilisée » où l'on ne vérifie plus les résultats comme l'explique F. François (1991). Ce stade de la recherche pouvant entrainer des pratiques figées. Pourtant, concernant la langue orale et son apprentissage, ce risque existe-t-il ? J.-F. Halté et M. Rispail (2005, p. 9) insistaient sur la nécessité d'y travailler sans répit: «Décrire, analyser sans répit les pratiques d'oral dans la classe (« soit que se passe-t-il dans la classe quand « ça » parle?») constitue une phase, insuffisante en elle-même, et en même temps totalement indispensable, du travail à mener sur ce territoire encore trop peu étudié. » Ce risque d'assèchement existe-t-il quand on sait l'unanimité des chercheurs concernant la difficulté à enseigner la langue orale ? Pour M. Laparra (2008, p. 119) :

«L'école est toujours en difficulté quand elle doit construire des apprentissages dont une partie des savoirs et savoir faire impliqués ont été acquis majoritairement hors d'elle. Cela est tout particulièrement vrai en ce qui concerne les apprentissages linguistiques et langagiers d'une manière générale, mais ce l'est plus encore en ce qui concerne les apprentissages oraux. " 
Enfin, le langage, à fortiori quand il est en cours d'acquisition, est un objet d'étude complexe pour lequel il est difficile de donner, dans le cadre de son apprentissage, des recettes. Dans ces conditions, peut-il réellement y avoir consensus? Dans tous les cas, il s'agit d'« analyser sans répit les pratiques d'oral » et de saisir la possibilité donnée de croiser les regards pour les faire évoluer. Bien que nous penchant sur un corpus nouveau, nous emprunterons à notre expérience préalable la méthodologie utilisée - que nous adapterons - à savoir: une analyse à triple entrées, interactionnelle, syntaxique et régulatrice. Nous adopterons pour ce faire le plan suivant: pour chacune des trois entrées, nous exposerons brièvement les référents théoriques d'appui, nous proposerons l'analyse correspondante d'extraits du corpus CLEA et mettrons en perspective, quand cela sera souhaitable dans une perspective contrastive, donc de mise en relief, des exemples d'analyse d'autres corpus.

\section{Analyse interactionnelle}

3 Pour les interactionnistes, les échanges sont toujours configurés dans le cours d'une action située, liée à des activités sociales concrètes. Ils soulignent ce faisant la nécessité d'accorder une attention minutieuse aux activités effectives, in situ, des acteurs telles qu'elles s'expriment, entre autres dans le déroulement conversationnel (Mondada \& Pekarek Doehler, 2000). Le corpus CLEA ne relève pas d'un échange conversationnel, l'on retiendra néanmoins à son sujet la définition de l'interaction donnée par P. Charaudeau et D. Maingueneau (2002, p. 318) : "C'est d'abord ce processus d'influences mutuelles qu'exercent les uns sur les autres les participants à l'échange communicatif ». Il s'agit d'étudier la construction dialoguée de narrations, là où émergent les compétences narratives des élèves assurant des fonctions dans l'interaction: «Étudier la narration orale oblige en effet à prendre en compte deux dimensions [...]: sa dimension interlocutive et dialogique puisqu'elle s'inscrit dans un dialogue même si elle est monologale, a fortiori si elle est construite à plusieurs; ses fonctions et enjeux dans l'interaction...» (Nonnon, 1999, p. 104). Par ailleurs, aussi bien L. Vygotski (1997), H. Wallon (1945) que L. Lentin (1973), J. S. Bruner (1996), B. Py (1989) et L. Mondada (1995) ont posé l'interaction langagière comme base explicative de l'acquisition. L'attention aux échanges en contexte nous amène pour ce sujet à poser la question du rapport entre leur nature et l'acquisition des élèves : «Le questionnement de la didactique s'ouvre de plein droit une fois admis que l'interaction n'est pas favorable a priori à l'acquisition, question largement débattue aujourd'hui. » (Canelas-Trevisi \& Thévenaz-Christen, 2002, p. 22).

4 L'analyse interactionnelle repose sur le repérage des énoncés langagiers et de leur nature au sein des interactions maitre-élève-élèves et montre comment les acquisitions se sont réalisées ou non, grâce aux modalités interactives mises en place.

5 Nous nous inspirons de l'analyse menée dans une recherche précédente (Charvy, 2009; 2011) sur un corpus présentant des interactions langagières maitre-élève(s) en maternelle, où nous avons repéré et répertorié par exemple les interventions selon qu'elles constituent des consignes, des interrogations directes, des reprises, des apports d'information (la liste n'est pas exhaustive). Cette typologie interactionnelle permettant de constater les effets des différents énoncés langagiers enseignants sur ceux des élèves.

6 Nous y avons montré que lorsque l'enseignant énonce des apports d'information et ajuste ses productions verbales à celles de l'élève de moyenne section, ce dernier peut y puiser 
les éléments qui lui sont nécessaires pour faire fonctionner son système langagier. Nous y avons également constaté que lorsque l'adulte accueille les énoncés de l'enfant comme variantes acceptables du texte et les reformule si nécessaire, il propose alors des schèmes sémanticosyntaxique créateurs ${ }^{1}$ qui permettent à l'enfant de passer des variantes qui sont les siennes à un moment donné à une variante parlée plus élaborée syntaxiquement. D'autre part, nous avons montré que le recours aux questions, ordres, consignes ne favorise pas la mise en fonctionnement du langage. C'est cette méthodologie et ces résultats que nous avons mis à l'épreuve du corpus CLEA.

\section{Le rôle (langagier ?) de l'enseignant}

7 Comment ne pas interroger la nature langagière du rôle de l'enseignant qui parle peu ; cette posture de retrait en termes d'énoncés langagiers, parce qu'elle est récurrente, est délibérée. Pour en conduire l'analyse, nous avons choisi arbitrairement la séance $1 \mathrm{du}$ corpus proposé. Nous avons pris néanmoins le parti de vérifier dans la séance 2 si les éléments d'analyse dégagés pour la première séance étaient corroborés.

Aussi, dans la séance 1 , nous avons pu repérer 17 interventions enseignantes sur une totalité de 77 interventions, soit $22 \%$.

Pour une interaction enregistrée dans le cadre de la recherche initiale mentionnée dans l'introduction, les interventions de l'enseignant étaient au nombre de 96 contre 16 pour l'élève. Nous constatons ici un rapport presque inversé: le temps de parole de l'enseignant étant de $83 \%$.

Nous avons également répertorié la totalité des interventions de l'enseignante dans le cadre de la séance 1 , voilà quelques exemples ?

Tableau 1. Interventions de l'enseignant, séance 1

\begin{tabular}{|c|c|c|c|c|}
\hline Séance & $\mathrm{N}^{\mathrm{o}}$ & Loc & Interventions & \\
\hline 1 & 1 & $M$ & $\begin{array}{l}\text { on va raconter ensemble/ } \\
\text { l'histoire dans l'ordre/ et on } \\
\text { essaie de ne rien oublier }\end{array}$ & consigne \\
\hline 1 & 12 & M & quand est-ce qu'il a dit ouf & interrogation directe \\
\hline 1 & 16 & M & qu'est-ce qui se passe après & interrogation directe \\
\hline 1 & 19 & M & $\begin{array}{l}\text { Sohaib a dit la sorcière est } \\
\text { partie dans les choux }\end{array}$ & \multirow{2}{*}{$\begin{array}{l}\text { énoncé épilinguistique: porte sur le } \\
\text { texte produit par l'un ou l'autre des } \\
\text { participants, ici, reprise sans modificatior } \\
\text { de la source } \\
\text { à valeur corrective }\end{array}$} \\
\hline 1 & 20 & Sohaib & non les chaussures & \\
\hline 1 & 23 & M & $\begin{array}{l}\text { Pierre il perd ses chaussures } \\
\text { dans les pommes de terre et } \\
\text { après qu'est-ce qu'il se passe }\end{array}$ & $\begin{array}{l}\text { apport d'information sur le thème de } \\
\text { l'interaction + interrogation directe }\end{array}$ \\
\hline
\end{tabular}




\begin{tabular}{|l|l|l|l|l|}
\hline 1 & 61 & M & $\begin{array}{l}\text { les enfants/ avant de vous lire } \\
\text { l'histoire je vous ai dit qu'il } \\
\text { faudrait tout me raconter }\end{array}$ & rappel de consignes \\
\hline
\end{tabular}

11 Les énoncés langagiers ici répertoriés sont constitués de 10 interrogations directes que nous nommerons questions, 5 consignes ou rappels de consignes, 1 conseil (qui ajuste et oriente la nature des échanges). En termes d'énoncés épilinguistiques qui portent sur le texte produit par l'un ou l'autre des participants, nous constatons également l'absence de reprise avec modification de la source ou de reformulation de la part de l'enseignant. Juste une concession dans ce domaine (sans modification de la source) à valeur corrective toutefois :

Tableau 2. Reprise de l'enseignant à valeur corrective

\begin{tabular}{|l|l|l|l|}
\hline Séance & $\mathbf{N}^{\mathbf{0}}$ & Loc & Intervention \\
\hline 1 & 19 & M & Sohaib a dit la sorcière est partie dans les choux \\
\hline
\end{tabular}

12 La séance 2 corrobore ces premiers résultats. L'enseignante pose 10 questions sur l'ensemble des interventions (rappel, 77 dans l'interaction sus-citée) : elles représentent seulement $12 \%$ des interventions. Il s'agit de questions uniques (au contraire de questions en rafale) et qui le plus souvent invitent à poursuivre le récit :

Tableau 3. Interventions de l'enseignant, séance 2

\begin{tabular}{|l|l|l|l|}
\hline Séances & $\mathbf{N}^{\mathbf{0}}$ & Loc & Interventions \\
\hline 1 & 16 & M & qu'est-ce qui se passe après \\
\hline 1 & 23 & M & $\begin{array}{l}\text { Pierre il perd ses chaussures dans les pommes de terre et après qu'est-ce } \\
\text { qu'il se passe }\end{array}$ \\
\hline 3 & 29 & M & oui/ Marianne \\
\hline 3 & 23 & M & Reda \\
\hline
\end{tabular}

Dans la séance 2, il est intéressant de constater un autre type de questionnement, portant sur l'implicite de l'histoire.

Tableau 4. Les questions de compréhension

\begin{tabular}{|l|l|l|l|}
\hline Séance & $\mathbf{N}^{\mathbf{0}}$ & Loc & Interventions \\
\hline 2 & 156 & Elise & il est un peu méchant// \\
\hline 2 & 157 & M & alors explique ce un peu méchant \\
\hline
\end{tabular}




\begin{tabular}{|l|l|l|l|}
\hline 2 & 160 & Sandra & et il défend son jardin \\
\hline 2 & 161 & M & $\begin{array}{l}\text { il défend son jardin qu'est-ce que vous auriez fait vous à la place de } \\
\text { monsieur Grégory/ Paulo }\end{array}$ \\
\hline
\end{tabular}

Nous rappelons que la consigne initiale posée par l'enseignant est celle-ci :

Tableau 5. La consigne initiale

\begin{tabular}{|l|l|l|l|}
\hline Séance & $\mathbf{N}^{\mathbf{0}}$ & Loc & Intervention \\
\hline 1 & 1 & $\mathrm{M}$ & $\begin{array}{l}\text { on va raconter ensemble/ l'histoire dans l'ordre/ et on essaie de ne rien } \\
\text { oublier }\end{array}$ \\
\hline
\end{tabular}

La question que l'on peut se poser ici, dans la mesure où les élèves expliquent la psychologie des personnages, est la suivante: font-ils bien le lien avec la consigne première ? Et de façon générale, l'enjeu de la séance, à savoir l'apprentissage langagier de la narration, est-il explicite pour les élèves? Des propositions de J.-P. Astolfi (1997) concernant le contrat didactique, nous retenons: le système d'obligations réciproques maitre-élève; un contrat "déjà-là » assorti à l'existence d'une situation didactique; le métier d'élève clarifié. Aussi la question posée de façon récurrente par les didacticiens : « Y a-t-il un contrat didactique quand il s'agit pour les enseignants d'apprendre à parler aux élèves? » reste entière. En d'autres termes, ces paramètres sont-ils réunis quand l'élève raconte une histoire, et à fortiori, qu'il essaie de dire les motivations internes des personnages?

En conclusion, l'enseignante ne présente pas d'offre langagière sur laquelle s'appuieraient les élèves. Les questions n'offrent pas à l'élève de « modèles ». Le rôle de l'adulte consiste à rappeler le cadre de l'activité, à permettre de rester dans l'échange, à réguler les prises de parole (notamment en sollicitant les plus petits parleurs), à inviter à poursuivre mais aussi à faire en sorte que les élèves se soutiennent du point de vue langagier :

Tableau 6. Soutien langagier entre élèves

\begin{tabular}{|l|l|l|l|}
\hline Séance & $\mathbf{N}^{\mathbf{0}}$ & Loc & Interventions \\
\hline 2 & 71 & $\mathrm{M}$ & attends elle va le dire/ vas-y Sima elle t'a un peu aidée \\
\hline 2 & 72 & Sima & \\
\hline 2 & 73 & M & redis-lui Sandra \\
\hline 2 & 74 & Sandra & il essaie d'enlever son gilet \\
\hline
\end{tabular}

17 Par ailleurs, nous notons la quasi-absence de feedback ou d'évaluation. 
Tableau 7. Absence de feedback enseignant

\begin{tabular}{|l|l|l|l|}
\hline Séance & $\mathbf{N}^{\mathbf{0}}$ & Loc & Interventions \\
\hline 2 & 317 & Elise & il s'est enfuyé \\
\hline 2 & 319 & M & il a failli se faire// dévorer \\
\hline
\end{tabular}

18 Le retrait de l'enseignant, en termes d'énoncés langagiers, a pour corollaire une prise de parole dense des élèves (qui occupe $78 \%$ de l'interaction).

\section{Interactions entre élèves}

19 Si les élèves occupent $78 \%$ de l'interaction, c'est là que l'essentiel se joue. Quelle analyse des interactions langagières des élèves? Nous avons choisi d'observer une succession d'interactions d'élèves entre deux interventions de l'enseignante de : S1-2 Mourad à S1-11 Oudriss. Selon la typologie utilisée, il s'agit à chaque fois d'apports d'information d'élèves différents (sept élèves au total) sauf pour Mourad, Shona et Oudriss, qui prennent deux fois la parole chacun. La question est de savoir si les élèves interagissent au sens où ils reprennent des éléments langagiers préalablement proposés par un ou plusieurs de leurs camarades.

Dans le paragraphe ci-dessous, nous avons attribué un même codage graphique (italique, caractère gras, police de caractère) aux reprises d'éléments identiques.

Mourad (S1-2) énonce la situation initiale (personnages + mise en garde maternelle) / Flora (S1-3) reprend les personnages et introduit l'élément perturbateur (monsieur Grégory) et parvient presque à la fin de l'histoire (la maman voit la preuve de la bêtise) / Shona (S1-4) complète la mise en garde (madame Grégory a fait du père lapin un civet) / Oudriss (S1-5) apport + /Pierre : " atchoum » + mise en garde /Sohaib (S1-6) apport + / Pierre : intrépide /Sihène (S1-7) apport +/monsieur Grégory et Pierre : course poursuite et fatigue / Daoud (S1-8) : apport +/mise en garde maternelle (reformulation) /Mourad (S1-9) reformulation mise en garde maternelle /Shona (S1-10) apport +/mise en garde maternelle/course-poursuite/chaussures dans les choux et les pommes de terre, gilet enlevé/monsieur Grégory(se retourne)/atchoum/remise /Oudriss (S1-11) prénoms (reprise) + ouf

Selon la typologie utilisée, il s'agit à chaque fois d'apports d'information d'élèves différents (sept élèves au total) sauf pour Mourad, Shona et Oudriss, qui prennent deux fois la parole chacun. Seuls deux élèves ne prennent pas la parole: Manolo (4 ans et 10 mois) et Beth (4 ans et 3 mois). Étant donné leur âge, on présume que ce sont des élèves de moyenne section. À ce titre, nous pouvons éventuellement interroger pour eux l'adaptation du dispositif. Sept élèves sur neuf prennent la parole dont trois, deux fois. Par rapport au choix du regroupement (groupe hétérogène de neuf élèves), le constat d'une prise de parole partagée et collective peut être posé. La question est de savoir si, en réalité, les élèves interagissent. On lit assez nettement un phénomène de reprise thématique quasi systématique : dans la prise de parole qui succède, une reprise de cette nature apparait. Mais dans le même temps, on note la complétion de deux façons: le même thème - repris - est augmenté, précisé : Mourad (S1-2) énonce la situation initiale ( 
personnages + mise en garde maternelle), Shona (S1-4) complète la mise en garde (madame Grégory a fait du père lapin un civet). D'autre part, chaque élève fait avancer le récit : Mourad (S1-2) énonce la situation initiale (personnages + mise en garde maternelle ), Flora (S1-3) reprend les personnages et introduit l'élément perturbateur (monsieur Grégory). Les élèves interagissent bel et bien malgré (ou grâce à) un enseignant en retrait.

\section{Analyse syntaxique}

Nous avons analysé la structuration syntaxique des narrations de chacun des élèves. Il s'est agi de repérer la présence de : silence(s) ; mot(s) ; syntagme(s) ; phrase(s) simple(s) ; phrase(s) simple(s) juxtaposée(s) ; phrase(s) simple(s) coordonnée(s) ; phrase(s) complexe (s).

Les critères retenus sont les suivants : la longueur d'énoncé ; la complexité syntaxique des énoncés de l'enseignant, de l'élève : pour ce qui concerne les élèves, on peut constater une organisation syntaxique de ces éléments et l'apparition d'introducteurs de complexité, d'abord isolés puis en combinatoire; les reprises d'un énoncé précédent. Il convient d'étudier le contexte sémanticosyntaxique: dans lequel les mots utilisés par l'enfant sont repris par un autre enfant, et dans lequel l'enfant reprend ou non les énoncés du texte-support.

Cette analyse permet de repérer la présence de phrases simples, de phrases simples multiples et de phrases complexes dans le langage de l'élève. Elle permet de repérer le degré de maitrise du langage explicite notamment en relevant les «introducteurs de complexité $»^{2}$, actualiseurs de la mise en relation des éléments d'une pensée capable de coordination et de synthèse.

\section{Nature syntaxique des énoncés et reprises}

Dans le cadre de l'analyse correspondante de la séance 1, ont été répertoriés tous les énoncés langagiers des élèves. Ils ont été ensuite classés selon leur nature syntaxique.

- Silence(s) : pour deux élèves

- Mot(s) : Flora (S1-53)

- Syntagme(s) : Mourad (S1-77)

- Phrase(s) simple(s): Sihène (S1-14) /Mourad (s1-18) /Oudriss (S1-25) /Shona (S1-22)/ Oudriss (s1-25)/ Flora (S1-28)/ Mourad (S1-30)/ Sohaib (S1-62)/Sohaib (S1-73)/Mourad (S1-74)

- Phrase(s) simple(s) juxtaposée(s) : Mourad (S1-26) /Shona (S1-52) /Oudriss (S1-65)

- Phrase(s) simple(s) coordonnée(s): Mourad (s1-2) /Oudriss (S1-5) /Sihèn (S1-7) /Daoud (S1-8) /Mourad (S1-9) /Oudriss (S1-11) /Oudriss (S1-13) /Mourad (S1-15) /Flora (S1-32) / Sohaib (S1-51) /Daoud (S1-69)

- Phrase(s) complexe(s) : Flora (s1-3) [parce que] /Shona (S1-4) [quand] /Sohaib (S1-17) [si... que] /Mourad (S1-21) [il croyait que] /Shona (S1-33) [qui (relatif)+ v. suivi d'un infinitif + quand] /Sohaib (S1-35) [parce que] /Mourad (S1-36) [parce que] /Sihène (S1-39) [quand, parce que, pour + infinitif] /Flora (S1-41) [pour + infinitif] /Daoud (S1-42) [quand] /Shona (S1-43) [relative] /Sihène (S1-44) [tellement que] /Mourad (S1-45) [quand] /Oudriss (S1-46) [quand] /Shona (S1-48) [quand, parce que] /Sihène (S1-49) [parce que, si] /Mourad (S1-50) [si] /Flora (S1-55) [parce que] /Mourad (S1-56) [que-subordination] /Shona (S1-66) [quand] / Shona (S1-72) [que-subordination] 

et reformulations sont considérées comme un des facteurs clés de l'appropriation des constructions de la langue. Les chercheurs en linguistique de l'acquisition s'inscrivant dans la lignée des travaux de L. Lentin s'intéressent à la dynamique de l'apprentissage. Ils cherchent à repérer une évolution dans le langage de chaque enfant et cherchent à évaluer les modalités des interactions favorables à cette évolution. Trois axes sont plus particulièrement explorés : le premier étant les reprises et reformulations d'un locuteur à l'autre, (les deux autres étant constitués d'une part des essais langagiers de l'enfant, d'autre part, des « schèmes sémantico-syntaxiques créateurs » (Cf. supra).

\section{Analyse syntaxique en lien au support écrit}

31 Nous avons pu constater dans la recherche antérieure citée que pour certains élèves, «ce sont les textes de l'album (quand la séance de langue orale porte sur la narration à partir de tels textes) qui, dans les deux interactions portant sur la narration d'album, servent d'offres langagières à Jeanne (une des élèves enregistrés) et non l'énoncé enseignant " (Charvy, 2009, p. 307). Il nous a semblé intéressant, par comparaison, de repérer si tel était le cas dans le cadre de ce corpus. 


\section{Flora ne reprend pas le texte de l'album mais le recompose.}

Tableau 8. Le texte de l'album recomposé

\begin{tabular}{|l|l|l|l|}
\hline Séance & $\mathbf{N}^{\mathbf{o}}$ & Loc & Intervention \\
\hline 1 & 3 & Flora & $\begin{array}{l}\text { eh bien Pierre/ Capucine et Neige et monsieur Grégory/ il veut les attraper/ } \\
\text { monsieur Grégory il veut attraper Pierre parce qu'il a faim/ comme ça sa } \\
\text { maman elle a vu ses chaussures/ et ses habits parce qu'il les avait enlevés/ } \\
\text { parce que monsieur Grégory il a voulu attraper Pierre }\end{array}$ \\
\hline
\end{tabular}

Il arrive également de façon assez explicite que le texte de l'album soit un appui, c'est le cas par exemple dans la séquence narrative 1 :

- «I I était une fois quatre petits lapins qui s'appelaient...» (texte de l'album)

- S1-2 Mourad : il y avait des lapins/ ils s'appelaient Romarin/ Capucine/ Neige/ Pierre/ et après leur maman dit à ses lapereaux/ méfiez-vous/ je dois m'absenter pour la journée

Ou la séquence narrative 2 :

- "Mes enfants, dit un jour madame Lapin, vous pouvez vous promener dans les champs ou le long du chemin, mais n'allez pas dans le jardin de monsieur Grégory. » (texte de l'album)

- «Votre père a eu un accident là-bas, madame Grégory en a fait un pâté (un civet). » (texte de l'album)

- S1-4 Shona : la maman elle a dit à ses petits lapins de ne pas y aller dans le potager parce que il y a monsieur Grégory/ elle a dit vous pouvez aller jouer dans le pré/mais surtout pas dans le potager parce que/ parce que dans le potager de monsieur Grégory votre pauvre père qui est allé/ il n'est jamais revenu et madame Grégory a fait un civet pour son repas

Par rapport au texte initial, voilà ce que l'on peut repérer dans la séquence narrative 2 :

- une reprise aménagée par transformation : discours direct $\rightarrow$ discours indirect?

- un aménagement du lien de causalité à l'aide de la conjonction de subordination "parce que $» ?$

- que certains élèves conservent (utilisent) le discours direct et n'ont pas recours à des phrases complexes.

Dans la séquence narrative 3 :

- une reprise aménagée par introduction de «Comme... »?

Dans la séquence narrative 4 :

- pas de reprise notable du texte littéraire initial.

Dans le récit de cet extrait par les élèves, nous ne repérons pas explicitement non plus de « reprises syntaxiques immédiates »... Aussi, l'album peut être un appui narratif, il est éventuellement un support d'offre lexicale mais il n'est pas un appui syntaxique.

\section{Le rôle des caractéristiques du support écrit}

Concernant ce corpus, il nous a été présenté le recours au texte support de la façon suivante: sur le plan didactique, il s'agit de choisir comme support de séance un texte narratif fictionnel non illustré que l'enseignant peut lire ou raconter. En l'occurrence, 
toutes les séances du corpus présenté portent sur un même support, le texte lu de Danger dans le potager.

Par comparaison, dans le cadre de l'apprendre à parler et de la mise en œuvre d'interactions langagières, nous avons choisi comme supports des textes narratifs illustrés répondant aux critères proposés par E. Canut (2006) : il faut qu'ils soient à la portée des enfants et comportent des variantes écrites « parlables » mais ils doivent aussi être explicites et structurés pour rapprocher le fonctionnement langagier de l'enfant des textes qu'il rencontrera lorsqu'il deviendra lecteur. Nous rappelons avoir repéré que c'était les textes de l'album (quand la séance de langue orale porte sur la narration à partir de tels textes) qui, dans les deux interactions portant sur la narration d'album, servaient d'offres langagières pour une élève et non l'énoncé enseignant. Or ici, le support écrit ne répond pas aux critères énoncés plus haut, pour autant les élèves énoncent des phrases complexes et leur prise de parole s'élève à $78 \%$ de l'ensemble de la séance 1 , par exemple.

\section{Analyse régulatrice}

Il nous reste à traiter un troisième axe d'analyse, le plus difficile sans doute à appréhender et à vérifier concrètement mais que la composante humaine, en termes de respect de l'élève, nous invite à le prendre en compte. Nous posons l'hypothèse que des caractéristiques propres aux pratiques langagières enseignantes, relevant de la régulation de l'interaction, sont facilitatrices pour l'acquisition de la langue orale.

La régulation de l'interaction repose sur la fonction de facilitation (rappel de l'album ou de l'activité, par exemple), le respect de la face (Goffman, 1974), le maintien des conditions de félicité, la ritualisation de la situation (Bruner, 1996), l'existence d'un contrat didactique (Brousseau, 2003).

Le projet qui nous est soumis à travers le corpus CLEA s'articule, en effet, autour de ce questionnement: comment l'école s'empare du langage de la famille et, à l'école, quels sont les rôles langagiers respectifs de l'élève et de l'enseignant au sein des interactions ?

En référence à E. Goffman (1974), cette définition de la situation par les acteurs provient du fait que l'interaction est un lieu de positionnement réciproque et de la construction des relations sociales. C'est ainsi que R. Vion (1992) interroge la notion de "rapport de places » liée à la notion de rôle, elle-même dépendante de la notion de statut.

R. Vion va encore plus loin en dessinant les contours de «l'espace interactif» déterminé par le fait que chaque sujet soit partie prenante dans la construction des places et donc de la relation.

La notion d'espace interactif permet d'appréhender la notion de face goffmanienne de manière plus complexe que comme une image homogène de soi. Voilà quelle définition E. Goffman (1974, p. 10) donne de la face:

«L'individu a généralement une réponse émotionnelle immédiate à la face que lui fait porter un contact avec les autres [...]. Si les évènements lui font porter une face plus favorable qu'il ne l'espérait, il "se sent bien". Si ses vœux habituels ne sont pas comblés, on s'attend à ce qu'il se sente "mal" ou "blessé". "

La face étant la valeur sociale positive qu'une personne revendique effectivement, mais, dit E. Goffman (ibid.: 15) : « garder la face est une condition de l'interaction et non son but». 


\section{Quelques premières traces d'une interaction régulée}

Nous prendrons tout d'abord l'exemple de la présence d'éléments de ritualisation de la situation (S2-231 ; S1-61). L'enseignant prend le soin de rappeler ce qui relève d'habitudes partagées, presque de rituels, ce qui concourt à la sécurité nécessaire à la participation confiante des élèves.

Un autre exemple tient à la présence de critères en lien avec ce que E. Goffman appelle le respect de la face. Nous trouvons par exemple dans le corpus, de la part de l'enseignant, des encouragements ou relances (S2-103; S2-63; S2-105). Mais aussi par exemple, des acquiescements (énoncés identifiés selon la typologie présentée, cf. supra.) qui permette que l'élève « se sente bien » (S2-3).

Enfin, on notera la présence d'une consigne forte (qui pourrait être : "Shona, tais-toi ») mais ici que l'enseignant prend soin d'édulcorer (S2-34).

Tableau 9. Éléments de ritualisation de la situation d'apprentissage, encouragements ou relances, acquiescements, injonction édulcorée

\begin{tabular}{|c|c|c|c|}
\hline Séances & $\mathrm{N}^{\mathrm{o}}$ & Loc & Interventions \\
\hline 2 & 231 & M & $\begin{array}{l}\text { et toi Paulo au moins tu vas faire une phrase// tu sais pour mon travail et } \\
\text { ensuite le tien j'ai besoin de savoir comment vous parlez j'écris des choses } \\
\text { mais pour ça il faut que tu parles/ c'est le travail d'aujourd'hui/ parler// si } \\
\text { tu parles pas du tout c'est dommage Paulo// }\end{array}$ \\
\hline 1 & 61 & M & $\begin{array}{l}\text { les enfants/ avant de vous lire l'histoire je vous ai dit qu'il faudrait tout me } \\
\text { raconter }\end{array}$ \\
\hline 2 & 103 & $\mathrm{M}$ & $\begin{array}{l}\text { si vous voulez l'aider lui donner un petit indice vous pouvez// Gaël tu } \\
\text { penses à quelque chose? aide-le un peu/// alors Kaïss/// Soliman/ il sort } \\
\text { de l'arrosoir et la course folle reprend// }\end{array}$ \\
\hline 2 & 63 & M & mais si je sais que vous savez moi/ on a le temps \\
\hline 2 & 105 & M & $\begin{array}{l}\text { laissez-lui un peu de temps vous savez qu'il faut lui laisser un peu de temps } \\
\text { à Soliman mais il va y arriver/ }\end{array}$ \\
\hline 2 & 3 & M & très bien// Alice \\
\hline 2 & 34 & M & Shona on va laisser parler un petit peu les autres \\
\hline
\end{tabular}

\section{Conclusion}

51 Le corpus proposé ne nous a pas permis de mener une analyse longitudinale or il est difficile de savoir si l'élève apprend à parler en maternelle sans faire porter le regard du chercheur sur un corpus au long cours.

52 L'intérêt ici était de croiser les regards entre chercheurs d'une part et d'accéder d'autre part à un corpus différent qui interroge à la fois une pratique de formateur d'enseignants 
et de chercheurs car comme le dit G. Bachelard $(1977$, p. 15) : «Il vient un temps où l'esprit aime mieux ce qui confirme son savoir que ce qui le contredit, où il aime mieux les réponses que les questions. Alors l'instinct conservatif domine, la croissance spirituelle s'arrête [...]». Car l'analyse en particulier interactionnel et syntaxique montre que l'enseignant dont la posture est « en retrait » laisse une place aux énoncés langagiers des élèves, et cette place les élèves la tiennent et la prennent.

Très brièvement, dans un souci de comparaison mais aussi de remise en cause d'un ouvrage qu'il faut sans cesse remettre sur l'établi, nous rappelons les conclusions de la recherche que nous avons menée et dont il a été question à plusieurs reprises dans cet article. Nous avons pu constater et vérifier dans notre recherche précédente que lorsque l'expert, l'enseignant en l'occurrence, énonce des apports d'information et ajuste ses productions verbales à celles de l'élève de moyenne section, ce dernier peut y puiser les éléments qui lui sont nécessaires pour faire fonctionner son système langagier. $\mathrm{Au}$ contraire du questionnement, il semble que lorsque l'adulte accueille les énoncés de l'enfant comme variantes acceptables du texte et les reformule si nécessaire, il propose alors des schèmes sémantico-syntaxiques créateurs qui permettent à l'enfant de passer des variantes qui sont les siennes à un moment donné à une variante parlée plus élaborée syntaxiquement. D'autre part, le recours aux questions, ordres, ne favorise pas la mise en fonctionnement du langage (Charvy, 2009).

Aussi, nous avons cru repérer certaines des conditions qui permettent l'allongement et la complexification syntaxique des énoncés de l'élève. Mais après avoir travaillé sur ce corpus, CLEA, et alors que nous avons fait, dans notre recherche initiale, un choix différent de celui de la pédagogie de l'écoute (Péroz, 2010), comment ne pas entendre que la voie empruntée n'est pas la seule? Non, il n'y a pas consensus au sujet de l'apprentissage du langage oral à l'école maternelle. Mais c'est sans doute ce que l'on peut espérer de mieux pour une recherche vivante.

Nous terminerons par une dernière question - que l'analyse du corpus CLEA n'élucide pas : que faire pour apprendre à parler à des élèves en difficulté, en particulier à des élèves allophones?

\section{BIBLIOGRAPHIE}

ASTOLFI, J.-P. (1997). L'erreur, un outil pour enseigner. Paris : ESF Éd.

BACHELARD, G. (1977) [1938]. La formation de l'esprit scientifique. Paris : Vrin.

BROUSSEAU, G. (2003). Glossaire de quelques concepts de la théorie des situations didactiques en mathématiques. En ligne : http://guy-brousseau.com/wp-content/uploads/2010/09/ Glossaire_V5.pdf.

BRUNER, J. S. (1996) [1983]. Le développement de l'enfant. Savoir faire, savoir dire. Trad. de l'anglais par M. Deleau. Paris : Presses universitaires de France. 
CANELAS-TREVISI, S. \& THÉVENAZ-CHRISTEN, T. (2002). «L'étude des interactions en classe de français langue étrangère et langue maternelle : deux "didactiques" au banc d'essai ? ». Revue française de pédagogie 141, p. 17-25. En ligne : https://www.persee.fr/doc/

rfp_0556-7807_2002_num_141_1_2911.

CANUT, E. (éd.) (2006). Apprentissage du langage oral et accès à l'écrit. Travailler avec un chercheur dans l'école. Amiens : Scérén/CRDP d'Amiens.

Charaudeau, P. \& maingueneau, D. (dirs) (2002). Dictionnaire d'analyse du discours. Paris : Seuil.

CHARVY, N. (2009). L'influence des pratiques langagières enseignantes sur l'acquisition de la langue orale chez les élèves entre quatre et cinq ans. Thèse : Université de la Sorbonne Nouvelle.

CHARVY, N. (2011). « La recherche comme aide à la conscientisation par l'enseignant de son "offre langagière” ». ELA. Études de linguistique appliquée 161, p. 37-44. En ligne : https://www.cairn.info/ revue-ela-2011-1-page-37.htm.

GOFFMAN, E. (1974) [1967]. Les rites d'interaction. Trad. de l'américain par Alain Kihm. Paris : Minuit. HALTÉ, J.-F. \& RISPAIL, M. (2005). L'oral dans la classe, compétence, enseignement, activités. Paris :

L'Harmattan.

LAPARRA, M. (2008). «L'oral, un enseignement impossible ? ». Pratiques 137-138, p. 117-134. En

ligne : https://journals.openedition.org/pratiques/1155.

LENTIN, L. (1973). «Interactions adultes-enfants au cours de l'acquisition du langage. Un exemple : étude contrastive du langage de deux enfants entre 3 et 4 ans et de celui de leurs parents (milieux socio-culturels différents) ». Études de linguistique appliquée 9, p. 9-50.

LENTIN, L. (2009) [1988]. Recherches sur l'acquisition du langage t. 2. Paris : Presses de la Sorbonne Nouvelle Paris 3.

MONDADA, L. (1995). « Analyser les interactions en classe : quelques enjeux théoriques et repères méthodologiques ». TRANEL 22, p. 55-89.

MONDADA, L. \& PEKAREK DOEHLER, S. (2000). «Interaction sociale et cognition située : quels modèles pour la recherche sur l'acquisition des langues?». AILE 12. En ligne : https:// journals.openedition.org/aile/947.

NONNON, É. (1999). «L'enseignement de l'oral et les interactions verbales en classe : champs de référence et problématiques. Aperçu des ressources en langue française ». Revue française de pédagogie 129, p. 87-131. En ligne : https://www.persee.fr/doc/

rfp_0556-7807_1999_num_129_1_1067.

PÉROZ, P. (2010). Apprentissage du langage oral à l'école maternelle : pour une pédagogie de l'écoute. Metz : CRDP de Lorraine.

Péroz, P. (2015). « Trois ans de recherche sur le langage oral à l'école maternelle. Résultats et perspectives », Colloque international, Pratiques et l'enseignement du français : bilan et perspectives, Université de Lorraine, Metz.

PY, B. (1989). «L'acquisition vue dans la perspective de l'interaction ». DRALV 41, p. 83-100.

VION, R. (1992). La communication verbale. Analyse des interactions. Paris : Hachette.

VYGoTSKI, L. (1997) [1934]. Pensée et langage. Trad. du russe par F. Sève. Paris : La Dispute.

WALlon, H. (1945). Les origines de la pensée chez l'enfant. Paris : Presses universitaires de France. 


\section{NOTES}

1. Les schèmes sémanticosyntaxiques créateurs ne fonctionnent pas comme des modèles à mémoriser et à reproduire. Ce sont « des stimulations mentales de mise en relation d'éléments verbalisables. Ce fonctionnement mental permet à l'apprenant, dans d'autres circonstances, de s'approprier pour son propre système langagier un fonctionnement dont on lui a donné l'expérience » (Lentin, 2009, p. 20).

2. Les Introducteurs de Complexité (IC) ont été déterminés expérimentalement au cours d'une enquête préliminaire à la recherche de L. Lentin, en 1969. La liste a été établie à partir d'un corpus de dialogues entre adultes et enfants âgés de 3 à 7 ans. Depuis la publication de la liste alphabétique originale en 1971, la liste des IC a connu quelques ajustements mais n'a pas été fondamentalement remise en cause. Au fil des années, la liste des IC présents dans des énoncés d'enfants entre 3 et 7 ans a été confirmée.

\section{RÉSUMÉS}

Cet article choisit de présenter une méthodologie reposant sur une analyse à triple entrées : interactionnelle, syntaxique et régulatrice du corpus CLEA. Nous empruntons à une expérimentation antérieure la méthodologie utilisée au sujet d'autres corpus que nous avons nous-même constitués. Aussi nous mettons en perspective l'analyse du corpus CLEA, dans une visée contrastive, donc de mise en relief, avec des résultats d'analyse de ces autres corpus. Malgré des modalités interactionnelles différentes (élèves-élèves au sein du corpus CLEA versus enseignant-élèves), des supports différents (texte narratif fictionnel non illustré versus album), nous posons le constat d'appropriations langagières avérées de part et d'autre.

This article is about a methodology relying on a three options analysis: a syntactic one, an interactive one, and a regulative of the CLEA corpus. We use parts of methodology which were used before about other corpus than the CLEA one, that we ourselves created and developed. As well, we can face the CLEA corpus analysis results relating to the other corpus analysis and have a parallel vision which give us the ability to see the contrasts, the differences but also gives us a global view of the project. In spite of the different interactive relations (pupils to pupils in the CLEA corpus vs. teacher to pupils), of the different media (non-illustrated narrative text vs. imagery books), we can affirm there is a real language appropriation in each part of the program.

\section{INDEX}

Mots-clés : interactions, nature syntaxique des énoncés, reprises, narration, support écrit, régulation enseignante

Keywords : interactive speech, syntactic utterance, repeats, narrative speech, written media, regulative teaching 


\section{AUTEUR}

NATHALIE CHARVY

Université de Bourgogne, Centre Interlangues - TIL, EA 4182, ESPÉ de Bourgogne, F-21000, France 